\title{
Local Contrast Enhancement using Local Standard Deviation
}

\author{
S. Somorjeet Singh \\ Department of Computer Science \\ Manipur University, Canchipur \\ H. Mamata Devi, PhD. \\ Assoc. Prof., Dept. of Computer Science \\ Manipur University, Canchipur
}

\author{
Th. Tangkeshwar Singh \\ Asst. Prof. (Sr. Scale), Dept. of Computer Science \\ Manipur University, Canchipur \\ Tejmani Sinam \\ Assoc. Prof., Dept. of Computer Science \\ Manipur University, Canchipur
}

\begin{abstract}
For a detailed visibility of an image, since only the global enhancement is not sufficient, local contrast enhancement plays a great role. One of the successful locally adaptive image contrast enhancement methods is by using Local Standard Deviation (LSD). The contrast enhancement using LSD is successfully used in many applications like in medical images, real time images, surveillance applications and many others. These types of applications employ a contrast factor which is divided by LSD. For such type of methods, limitation occurs when the LSD becomes zero. This limitation due to divide by zero can be overcome by adjusting the LSD to a modified value.
\end{abstract}

\section{Keywords}

Local contrast enhancement, local standard deviation, divideby-zero, removal.

\section{INTRODUCTION}

People love good quality pictures or images. However, all the pictures or images are not always having good quality depending on various factors like recording, recording device, light incident on the objects and processing etc. One of the low quality images is the low contrast image type. Low contrast images are having narrow range of intensity levels of the pixels of the image. To improve the visual quality of low contrast images, different enhancement can be done in different domain like spatial domain, frequency domain etc.

Global contrast enhancement techniques [1]-[5] work fine, fast and simple for some kind of images. It improves the quality of the image globally, however, the quality of the local contrast is poor in some images. One of the widely utilized global enhancement methods is histogram equalization. Global Histogram Equalization (GHE) is very effective and the most appropriate enhancement method for some images. However, GHE is not always desirable [6], because it produces the image over enhanced in some portions and some properties of the image cannot be defined properly.

Since the image characteristics differ considerably from one region to another in the same image, the enhancement techniques based on local contrast are necessary. There are several local contrast enhancement methods. Dorst [7] adapted histogram stretching method over a neighbourhood around the candidate pixel for local contrast stretching, and followed by a numerous modifications [8]-[10] of histogram equalization based on adapting the same over a sub-region of the image. A local contrast stretching method as suggested by Lee [11],[12] makes use of local statistics of a predefined neighbourhood in modifying the gray level of a pixel. Narendra and Fitch [13] designed the amplification factor too to be a function of the pixel based on the local gray level statistics over the same neighbourhood in which contrast gain is inversely proportional to LSD.

Dah-Chung [14] observed that image enhancement with contrast gain which is constant or inversely proportional to the LSD produces either ringing artifacts or noise over enhancement due to the use of too large contrast gains in regions with high and low activities, and developed a new method in which gain is a non-linear function of LSD and observed that this method is effective for medical images. Schutte [15] introduced a multi-window extension of this technique and showed how the window sizes should be chosen. Sascha [16] implemented an improved multi-window real-time high frequency enhancement scheme based on LSD in which gain is a non-linear function of the detail energy and observed that this method is effective for the surveillance and many other applications.

The local contrast enhancement using LSD is powerful in many applications. However, for some kind of images, the pixels of some regions of the image are producing the LSD's as 0's. This is an undesired limitation that divide by zero may not be working or may produce undesired output image.

This paper is organized as follows: Section 2 briefly reviews some methods based on LSD. Section 3 describes our proposed technique on removal of divide by zero. Section 4 shows experimental results and discussion. And Section 5 is for conclusion

\section{REVIEW ON SOME METHODS BASED ON LOCAL STANDARD DEVIATION}

In this section, we review some methods in which LSD's are employed.

First, one of the most frequently referred methods is Narendra and Fitch's [13] method. They presented a method using LSD information as

$$
f(i, j)=m_{x}(i, j)+\frac{D}{\sigma_{x}(i, j)}\left[x(i, j)-m_{x}(i, j)\right]
$$


where $\boldsymbol{x}(\boldsymbol{i}, \boldsymbol{j})$ is the gray scale value of a pixel in an image, $\boldsymbol{f}(\boldsymbol{i}, \boldsymbol{j})$ is the enhanced value of $\boldsymbol{x}(\boldsymbol{i}, \boldsymbol{j}), \boldsymbol{m}_{x}(\boldsymbol{i}, \boldsymbol{j})$ is the local mean, $\sigma_{x}(i, j)$ is the LSD and $\boldsymbol{D}$ is a constant. Here, contrast gain itself is spatially adaptive and inversely proportional to LSD.

Next is also one of the most frequently referred methods. DahChung [14] presented another method using LSD information as

$$
f(i, j)=m_{x}(i, j)+k(i, j)\left[\frac{x(i, j)-m_{x}(i, j)}{\sigma_{x}(i, j)}\right]
$$

where $\boldsymbol{k}(\boldsymbol{i}, \boldsymbol{j})$ is the contrast gain. This was applied on medical images.

Next is the Multi-Scale Adaptive Gain Control method of Schutte [15]. He described the enhanced output image as

$o=i+\sum_{j=1}^{k} M \frac{C_{j}}{L S D_{j}} \cdot\left(i-m_{j}\right)$

where, $\boldsymbol{o}$ is the output image, $\boldsymbol{i}$ is the input image, $\mathrm{k}$ is the number of scales used, $\boldsymbol{M}$ is the global mean of the image, $\boldsymbol{m}_{\boldsymbol{j}}$ is the local mean of the kernel, $\boldsymbol{C}_{\boldsymbol{j}}$ is the gain factor that control the enhancement level per kernel and $\boldsymbol{L} \boldsymbol{S} \boldsymbol{D}_{\boldsymbol{j}}$ is the local standard deviation per kernel.

Sascha [16] presented another improved method in which frequency bands are separately controlled and enhanced. This is achieved by subtracting windows of two subsequent scales so that the difference between windows represents a supplementary frequency band which is added to a certain pixel neighbourhood and which is additionally enhanced by the use of the bigger kernel:

$$
o=i+\sum_{j=1}^{k} M \frac{C_{j}}{L S D_{j}} \cdot\left(m_{j}-m_{j+1}\right)
$$

In all of the methods, (1)-(4), there employed a factor which is divided by the LSD. They are applied for different fields like medical images, real-time imaging, surveillance applications and many other applications. However, they may not be working or may produce undesired output image for some images in which LSD's of some pixels of the image are zero.

\section{REMOVAL OF DIVIDE BY ZERO CONDITION}

In the above equations (1)-(4), if LSD's of some of the pixels of the image are having the value zero, divide by zero is occurred. This divide by zero can be removed by increasing the window size or by modifying the values of LSD's of the image.

Although we can verify the removal of divide by zero condition in any of the above equations (1)-(4) or any similar equations, let us take the following method, for the simplicity, for verification:

$$
f(i, j)=x(i, j)+\frac{C}{\sigma(i, j)} \cdot[x(i, j)-m(i, j)]
$$

where, $x(i, j)$ is the gray scale value of a pixel in an image, $f(i, j)$ is the enhanced value of $\boldsymbol{x}(\boldsymbol{i}, \boldsymbol{j}), \boldsymbol{m}(\boldsymbol{i}, \boldsymbol{j})$ is the local mean, $\sigma(\boldsymbol{i}, \boldsymbol{j})$ is the LSD and $\boldsymbol{C}$ is a constant for contrast control.

Now, the two alternative techniques can be considered for the removal of divide by zero condition. First, it can be removed by increasing the size of the window because LSD's are zero when all the gray scale values of the pixels under the window are equal.

If the size of the window is taken larger, the chances for the gray scale values of the pixels under the window to be equal are less.

Secondly, each of LSD can be modified by adding a very small value which is greater than zero and a negligible value too. Now, equation (5) can be improved as

$$
f(i, j)=x(i, j)+\frac{C}{\sigma(i, j)+s} \cdot[x(i, j)-m(i, j)]
$$

where, $s$ is a very small and negligible quantity greater than zero. When $s$ is very small, equation (6) maintains the same quality of equation (5) except it removes the divide by zero condition for an output image. Human perception is no difference between the output images produced by the equations (5) and (6) of the same input image, except the divide by zero portions of the image, because human eye cannot detect the difference at the micro level or lower level for any intensity value of a pixel.

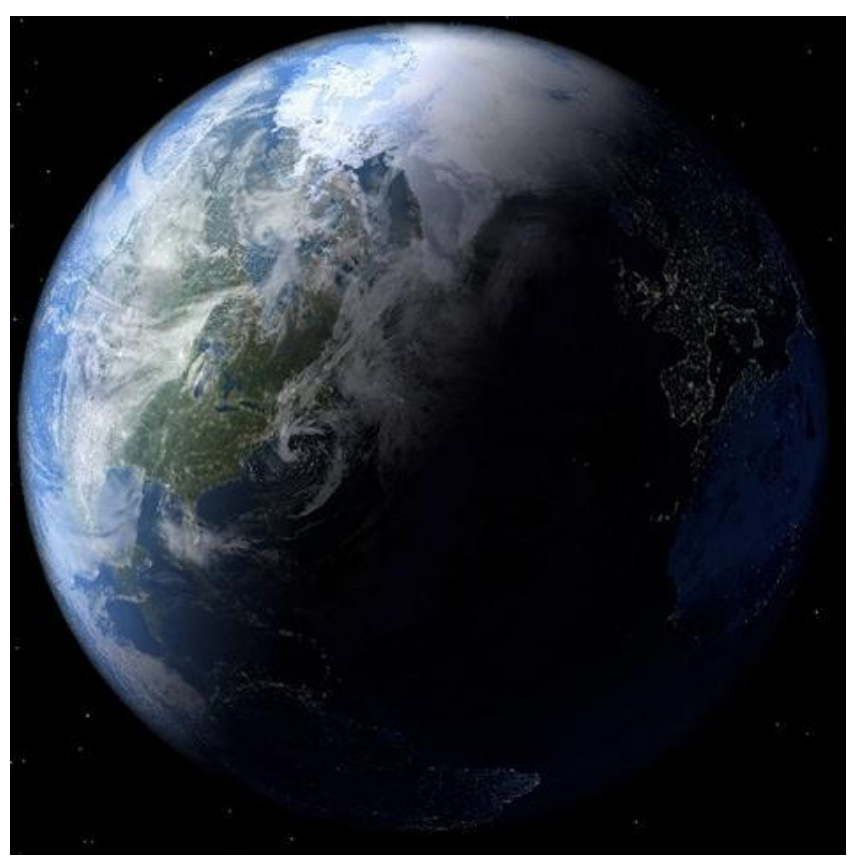

Fig. 1. Photograph of earth from space

Here, if we consider the quality of the enhancement, the first method may be changing in the quality of the enhancement because the increase in the window size makes a change in LSD's and local means so that there is no uniformity in the changing values for each pixel. This may lead to an undesirable image enhancement quality. And also, the choosing of the window size is dependent on the particular image so that how much equal gray values of contiguous pixels are present in the image. On the other hand, we can choose any desired size of the 
window for the second method i.e., equation (6). Hence, the second technique is the better method.

\section{EXPERIMENTAL RESULT AND DISCUSSION}

Table 1. No. of LSD with zero values against the window

\begin{tabular}{|c|c|}
\hline $\begin{array}{l}\text { Size of Window } \\
\text { (One side each) }\end{array}$ & No. of LSD with 0 value \\
\hline 3 & 62275 \\
\hline 5 & 49898 \\
\hline 7 & 39707 \\
\hline 9 & 35159 \\
\hline 11 & 34038 \\
\hline 13 & 30648 \\
\hline 15 & 27517 \\
\hline 17 & 24771 \\
\hline 19 & 22712 \\
\hline 21 & 20783 \\
\hline 23 & 18898 \\
\hline 25 & 17121 \\
\hline 27 & 15519 \\
\hline 29 & 14017 \\
\hline 31 & 12568 \\
\hline 35 & 10106 \\
\hline 55 & 3765 \\
\hline 75 & 1659 \\
\hline 85 & 1133 \\
\hline 95 & 732 \\
\hline 105 & 397 \\
\hline 115 & 133 \\
\hline 117 & 90 \\
\hline 119 & 49 \\
\hline 121 & 15 \\
\hline 123 & 0 \\
\hline 125 & 0 \\
\hline
\end{tabular}

We have tested the above two techniques for the removal of divide by zero condition in MATLAB 7.13.0.564 with different window sizes. A detailed study is performed on a satellite image, i.e., the photograph of earth taken from the space as in Fig. 1. First, we have counted the number of LSD's having the value zero against the relevant window sizes. Table 1 shows the relevant information with different window sizes. When the size of the window is small, number of LSD's with zero value is larger. If the size of the window is increased, the number of LSD's with zero value is smaller.

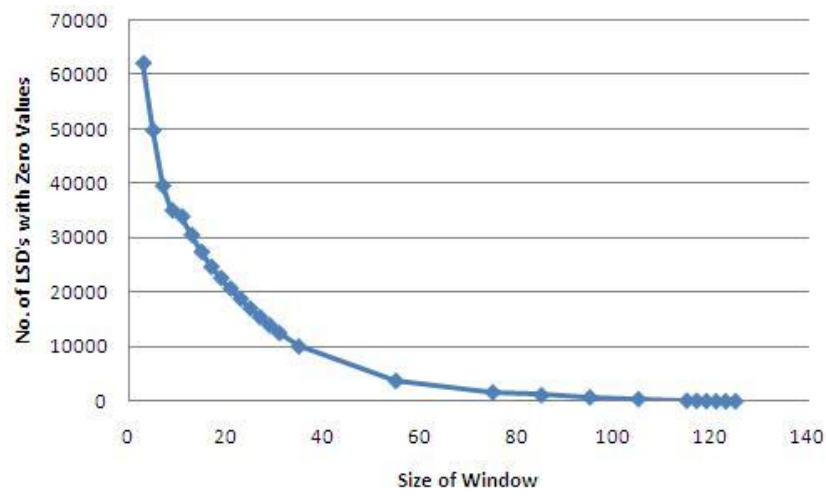

Fig. 2. Graph showing No. of LSD's with zero values against the Size of Window

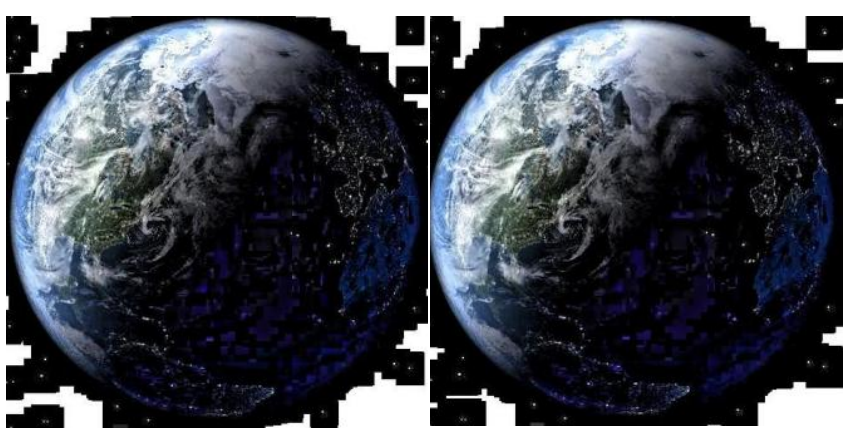

(a) With window size $25 \times 25$

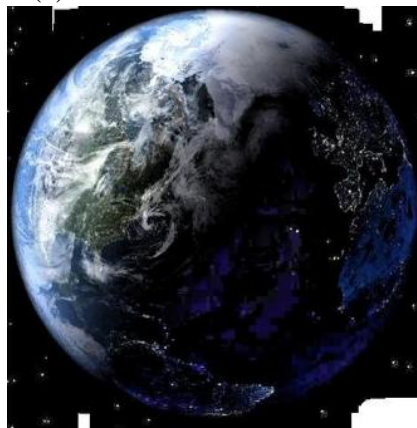

(b) With window size $35 \times 35$

(c) With window size $55 \times 55$

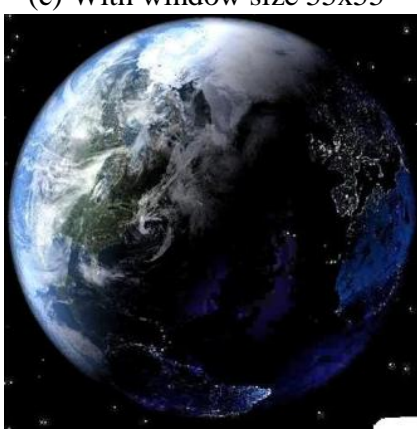

(e) With window size $95 \times 95$

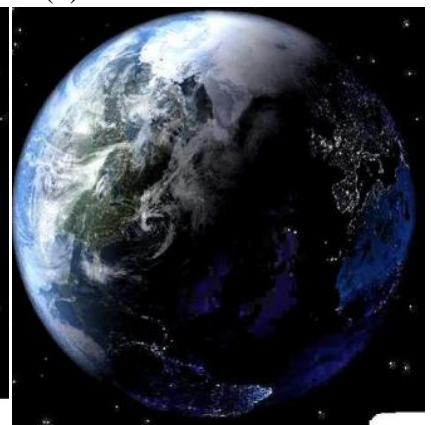

(d) With window size $85 \times 85$

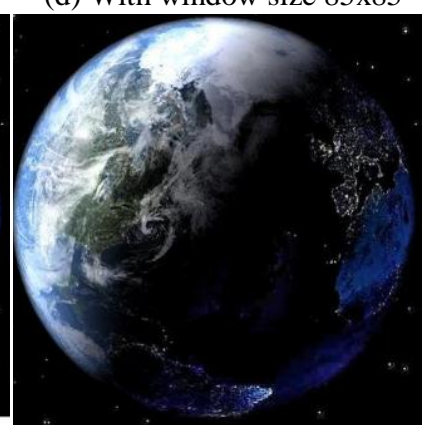

(f) With window size $125 \times 125$ Fig.3. Test images with different window sizes using eqn. (5)

When the size of the window is equal to $123 \times 123$ or greater than $123 \times 123$, there is no LSD with the value zero. The relation between the size of the window and the number of LSD's with zero values is illustrated in the graph as shown in Fig. 2.

Next, we have applied the equation (5) with different window sizes on the image, shown in Fig. 1, by taking the same $\boldsymbol{C}$ value. Fig.3. shows different images resulting from different window sizes. When the window size is small, in the dark portions of the image, larger sizes of the undesired effect, produced by 
divide by zero condition, are shown. When the window size is increased, the size of undesired effect due to divide by zero is reduced. And no undesired effect due to divide by zero found when window size is $125 \times 125$. However, the enhancement quality is reduced in increasing the window size too much. The first technique is, therefore, limited to the nature of the image.

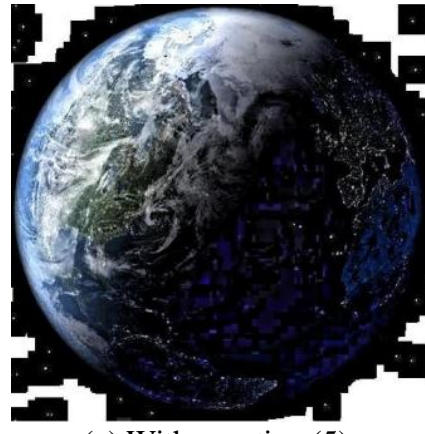

(a) With equation (5), Window size $=25 \times 25$

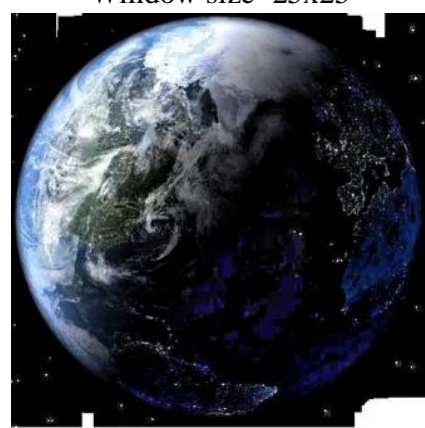

(c) With equation (5),

Window size $=55 \times 55$

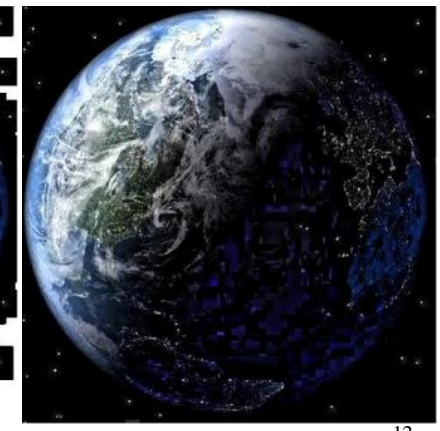

(b) With eqn. (6), $\mathrm{s}=1.0 \times 10^{-12}$ Window size $=25 \times 25$

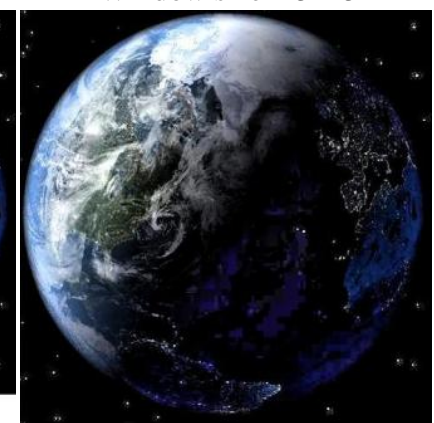

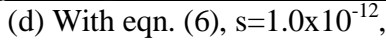
Window size $=55 \times 55$
Fig.4. Comparative Results with $\mathrm{C}=0.1$ (Using HSI format)

Table 2. Comparative Statements of Our Proposed Technique with Other Methods

\begin{tabular}{|c|c|}
\hline Methods of Equations (1)-(4) & $\begin{array}{c}\text { With Our } \\
\text { Proposed } \\
\text { Technique }\end{array}$ \\
\hline $\begin{array}{c}\text { If the LSD's of the pixels are zero, } \\
\text { undesired similar effect due to divide } \\
\text { by zero conditions are produced with } \\
\text { these methods as shown in fig. 3(a)- } \\
\text { (e), fig. } \mathbf{4}(\mathbf{a}) \text { and fig. }\end{array}$ & $\begin{array}{c}\text { No }(\mathbf{c}) \text {. } \\
\text { effect due to divide } \\
\text { by zero conditions } \\
\text { are produced. }\end{array}$ \\
$\begin{array}{c}\text { To control the undesired effect due to } \\
\text { divide by zero conditions, the size of } \\
\text { the window is to be increased up to a } \\
\text { certain amount, however, it may reduce } \\
\text { the quality of the enhancement }\end{array}$ & $\begin{array}{c}\text { Any desired size of } \\
\text { the window can be } \\
\text { taken without any } \\
\text { undesired effect due } \\
\text { to divide by zero } \\
\text { conditions. }\end{array}$ \\
\hline
\end{tabular}

When we examined with the second method, i.e., equation (6), any size of window can be taken as our desire. If we desire the window size to be $25 \times 25$, a comparison for equations (5) and (6) is shown in Fig. 4 (a) and (b). If we desire the window size to be $55 \times 55$, the difference of equations (5) and (6) is shown in Fig. 4 (c) and (d). Here, the value of $\boldsymbol{C}$ is taken as 0.1 and the value of $s$ for equation (6) is taken as 0.000000000001 , i.e., $1.0 \times 10^{-12}$ which is a negligible quantity. From these experiments, we have found that there is no difference between the quality in the enhancement of equations (5) and (6), except the divide by zero portions in the image. This means that divide by zero can be overcome easily with the technique of equation (6) at any desired level of window size.
The distinctive features of the proposed technique are shown in the comparative statements with equations (1)-(4), given in the Table 2.

\section{CONCLUSION}

Local Contrast Enhancement using LSD is widely used in different fields like Medical imaging, Real-Time Imaging, Surveillance and Security Applications and many others. Many people working with LSD for Local Contrast Enhancement may be suffering from divide by zero. This paper presents the removal techniques of the divide by zero effect without changing the enhancement quality. This paper will be beneficial to all who are interested on Local Contrast Enhancement using LSD.

\section{REFERENCES}

[1] S. Somorjeet Singh, Dr. H. Mamata Devi, Th. Tangkeshwar Singh, O. Imocha Singh, "A New Easy Method of Enhancement of Low Contrast Image Using Spatial Domain," International Journal of Computer Applications (0975 - 8887) Volume 40- No.1, February 2012

[2] R. C. Gonzalez and R. E. Woods, Digital Image Processing. $3^{\text {rd }}$ edition, Prentice Hall, 2008.

[3] Sascha D. Cvetkovic and Peter H. N. de With, "Image enhancement circuit using non-linear processing curve and constrained histogram range equalization," Proc. of SPIEIS\&T Electronic Imaging, Vol. 5308, pp. 1106-1116 (2004).

[4] M.A. Yousuf, M.R.H. Rakib, "An Effective Image Contrast Enhancement Method Using Global Histogram Equalization," Journal of Scientific Research, Vol. 3, No. 1, 2011.

[5] I. Altas, J. Louis, and J. Belward, "A variational approach to the radiometric enhancement of digital imagery," IEEE Trans. Image Processing, vol. 4, pp. 845-849, June 1995.

[6] T.-L. Ji, M. K. Sundareshan, and H. Roehrig, "Adaptive image contrast enhancement based on human visual properties," IEEE trans. Med. Imag., vol. 13, pp. 573-586, Aug. 1994.

[7] L. Dorst, "A local contrast enhancement filter," In Proc. 6th Int. Conference on Pattern recognition, pages 604-606, Munich, Germany, 1982.

[8] D. Mukherjee and B. N. Chatterji, "Adaptive neighborhood extended contrast enhancement and its modification," Graphical Models and Image Processing, 57:254-265, 1995.

[9] R. B. Paranjape, W. M. Morrow, and R. M. Rangayyan, "Adaptive-neighborhood histogram equalization for image enhancement," Graphical Models and Image Processing, 54:259-267, 1992.

[10] S. M. Pizer, E. P. Amburn, J. D. Austin, R. Cromartie, A. Geselowitz, T. Geer, B. H. Romeny, J. B. Zimmerman, and K. Zuiderveld, "Adaptive histogram modification and its variation," Computer Vision, Graphics and Image Processing, 39:355-368, 1987.

[11] J. S. Lee, "Digital image enhancement and noise filtering by use of local statistics," IEEE Trans. on Pattern Analysis and Machine Intelligence, PAMI-2:165-, 1980. 
[12] J. S. Lee, "Refined filtering of image noise using local statistics," Computer Graphics and Image Processing, 15:380-, 1981.

[13] P.M. Narendra and R.C. Fitch, "Real-time adaptive contrast enhancement," IEEE Trans. PAMI, Vol.3, no. 6, pp.655-661, 1981

[14] D.-C. Chang and W.-R. Wu, "Image contrast enhancement based on a histogram transformation of local standard deviation," IEEE Trans. MI, vol. 17, no. 4, pp. 518-531, Aug. 1998

[15] K. Schutte, "Multi-Scale Adaptive Gain Control of IR Images," Infrared Technology and Applications XXIII, Proceedings of SPIE Vol. 3061, pp.906-914 (1997).

[16] Sascha D. Cvetkovic, Johan Schirris and Peter H. N. de With, "Locally-Adaptive Image Contrast Enhancement Without Noise And Ringing Artifacts,” IEEE, ICIP 2007. 